\title{
Total Variation-Based Speckle Reduction Using Multi-grid Algorithm for Ultrasound Images
}

\author{
Chen Sheng 1 , Yang Xin ${ }^{1}$, Yao Liping ${ }^{2}$, and Sun Kun² \\ ${ }^{1}$ Institution of Image Processing and Pattern Recognition, \\ Shanghai Jiaotong University, Shanghai, P.R. China \\ chnshn@hotmail.com, yangxinasjtu.edu.cn \\ ${ }^{2}$ Shanghai Children's Medical Center, Shanghai Second Medical University, \\ Shanghai, P.R. China
}

\begin{abstract}
This paper presents an approach for speckle reduction and coherence enhancement of ultrasound images based on total variation (TV) minimization. The proposed method can preserve information associated with resolved object structures while reducing the speckle noise. However, since the equation system deduced by the TV-based method is a strongly nonlinear partial differential equation (PDE) system, the convergence rate is very slow when using standard numerical optimization techniques. So in this paper, we introduce the nonlinear multi-grid algorithm to solve this system. Numerical results indicate that the image can be recovered with satisfied result even contamination of strong noise using the proposed method and the algorithm of nonlinear multi-grid has more efficiency than the conventional numerical techniques such as conjugate gradient (CG).
\end{abstract}

\section{Introduction}

The low cost, portability, and real-time image formation make ultrasound imaging an essential tool for medical diagnosis. While ultrasound images often suffer from a special kind of noise called speckle. Speckle significantly degrades the image quality and affects human interpretation of the images as well as the accuracy of computerassisted diagnostic techniques. So a number of methods have been proposed to address the problem of speckle reduction such as temporal averaging [1], adaptive weighted median filtering (AWMF) [2], adaptive speckle reduction [3], Wiener filtering and wavelet shrinkage [4]. While these approaches could not succeed to balance between speckle suppression and feature preservation due to the complexity of speckle statistics.

In this paper, we describe a speckle reduction technique whereby the ultrasound image is smoothed to suppress the speckle while substantially preserving image components corresponding to resolved object structures. This technique is based on total variation (TV) minimization [5]. Due to its anisotropy, the proposed technique allows coherent structure enhancement while the dynamic smoothing is controlled by the local behavior of the images. In addition, to ensure real-time implementation, we apply nonlinear multi-grid method to solve the nonlinear TV-based minimization problem. 


\section{Methods}

Due to the limited dynamic range of commercial display monitors, ultrasound imaging systems compress the echo signal to fit in the display range. Such compression changes the characteristics of the signal probability density function (PDF). In particular, it affects the high intensity tail of the Rayleigh and Rician PDFs more than the low intensity part. As a result, the speckle noise becomes very close to white Gaussian noise corresponding the uncompressed Rayleigh signal [6]. The statistical properties of speckle noise were studied in [7]. It showed that, when the imaging system has a resolution cell that is small in relation to the spatial detail in the object, and the speckle-degraded image has been sampled coarsely enough that degradation at any pixel can be assumed to be independent of the degradation at all other pixels, coherent speckle noise can be modeled as multiplicative noise. Thus we have:

$$
f(x, y)=g(x, y) \eta_{m}(x, y)+\eta_{a}(x, y)
$$

where: $g(x, y)$ is an unknown piecewise constant two-dimensional function representing the noise-free original image, $f(x, y)$ is the noisy observation of $g(x, y)$, $\eta_{m}$ and $\eta_{a}$ are multiplicative and additive noise respectively, and $x$ and $y$ are variables of spatial locations that belong to 2-D space of all real numbers, $(x, y) \in R^{2}$. Since the effect of additive noise is considerably small compared with that of multiplicative noise, Equation (1) can be rewritten as:

$$
f(x, y)=g(x, y) \eta_{m}(x, y)
$$

The logarithmic amplification transforms the Equation (2) into the classical additive noise form:

$$
\log (f(x, y))=\log (g(x, y))+\log \left(\eta_{m}(x, y)\right)
$$

The above expression can be rewritten as

$$
z(x, y)=u(x, y)+\eta(x, y)
$$

At this stage, we can consider $\eta(x, y)$ to be white Gaussian noise and apply a conventional additive noise suppression technique, such as Wiener filtering. It is to find $u(x, y)$ which minimizes the functional:

$$
T(u)=\frac{1}{2}\|u-z\|^{2}+\alpha J(u)
$$

Common choices for $J$ are

$$
J(u)=\int u^{2} d x
$$

Equation (6) often induces blur in images and spurious oscillations when $u$ is discontinuous. 
So we consider the nonlinear TV functional:

$$
J_{T V}(u)=\int_{\Omega}|\nabla u| d x
$$

where: $\nabla u$ denotes the gradient of $u$ :

$$
\nabla u=\left(\frac{\partial u}{\partial x}, \frac{\partial u}{\partial y}\right)
$$

here: $u$ is not required to be continuous.

How ever, the Euclidean norm is not differentiable at zero. To avoid difficulties associated with the non-differentiability, the modification [8]:

$$
J_{\beta}(u)=\int_{\Omega} \sqrt{|\nabla u|^{2}+\beta^{2}} d x
$$

will be utilized here. Throughout the remainder of this paper, the functional to be minimized is:

$$
T(u)=\frac{1}{2}\|u-z\|^{2}+\alpha \int_{\Omega} \sqrt{|\nabla u|^{2}+\beta^{2}} d x
$$

The Eular-lagrange equation associates with Equation (8) is

$$
\begin{aligned}
& u+\alpha L(u) u=z, x \in \Omega \\
& \frac{\partial u}{\partial n}=0, x \in \partial \Omega
\end{aligned}
$$

where: $L(u)$ is differential operator whose action on $u$ is given by:

$$
L(u) u=-\nabla \cdot\left(\frac{1}{\sqrt{|\nabla u|^{2}+\beta^{2}}} \nabla u\right)
$$

It is an elliptic nonlinear PDE. From Equation (10), we can see that the smoothing decreases as the gradient strength increases and the smoothing is stopped across edges.

\section{Numerical Solutions}

There are many standard numerical optimization techniques such as CG method. However, these standard methods tend to perform poorly on TV minimization problems. The underlying difficulty is that the local quadratic approximation that is the basis for standard CG method is not a good model for the nonlinear TV-based minimization. So, in this paper we adopt the nonlinear multi-grid method to deal with this problem.

Multi-grid method was firstly introduced by Brandt [9]. Unlike the conventional methods, the multi-grid algorithm can solve nonlinear elliptic PDE with non-constant coefficients with hardly any loss in efficiency. In addition, no nonlinear equations need be solved, except on the coarsest grid. 
Suppose we discrete the nonlinear elliptic PDE of Equation (9) on a uniform grid with mesh size $h$ :

$$
T_{h}\left(u_{h}\right)=z_{h}
$$

where: $T_{h}\left(u_{h}\right)$ denote $u_{h}+\alpha L_{h}\left(u_{h}\right) u_{h}$.

Let $\tilde{u_{h}}$ denote some approximate solution and $u_{h}$ denote the exact solution to Equation (11). Then the correction is:

$$
v_{h}=u_{h}-\tilde{u_{h}}
$$

The residual is:

$$
T_{h}\left(\tilde{u_{h}}+v_{h}\right)-T_{h}\left(\tilde{u_{h}}\right)=f_{h}-T_{h}\left(\tilde{u_{h}}\right)=-d_{h}
$$

Now, we form the appropriate approximation $T_{H}$ of $T_{h}$ on a coarser grid with mesh size $H$ (we will always take $H=2 h$ ). The residual equation is now approximated by:

$$
T_{H}\left(u_{H}\right)-T_{H}(\tilde{u})=-d_{H}
$$

Since $T_{H}$ has smaller dimension, this equation will be easier to solve. To define $u_{H}$ and $d_{H}$ on the coarse grid, we need a restriction operator $R$ that restricts $\tilde{u_{h}}$ and $d_{h}$ to the coarse grid. That is, we solve:

$$
T_{H}\left(u_{H}\right)=T_{H}\left(R \tilde{u_{h}}\right)-R d_{h}
$$

on the coarse gird. Then the coarse-grid correction is:

$$
\tilde{v_{H}}=u_{H}-R \tilde{u_{h}}
$$

Once we have a solution $\tilde{v}_{H}$ on the coarse gird, we need a prolongation operator $P$ that interpolates the correction to the fine gird:

So we have:

$$
\tilde{v}_{h}=P \tilde{v}_{H}
$$

$$
\tilde{u}_{h}^{\text {new }}=\tilde{u}_{h}+P \tilde{v}_{H}
$$

It is the two-grid algorithm and can be easily extended to multi-grid.

The symbol of $P$ is found by considering $v_{H}$ to be 1 at some mesh point $(x, y)$, zero elsewhere, and then asking for the values of $P v_{H}$. The most popular prolongation operator is simple bilinear interpolation. It gives nonzero values at the 9 points $(x, y),(x+h, y), \ldots,(x-h, y-h)$ and its symbol is: 


$$
\left[\begin{array}{ccc}
\frac{1}{4} & \frac{1}{2} & \frac{1}{4} \\
\frac{1}{2} & 1 & \frac{1}{2} \\
\frac{1}{4} & \frac{1}{2} & \frac{1}{4}
\end{array}\right]
$$

The symbol of $R$ is defined by considering $v_{h}$ to be defined everywhere on the fine grid, and then asking what is $R v_{h}$ at $(x, y)$ as a linear combination of these values. The choice for $R$ is the adjoint operator to $P$. So that the symbol of $R$ is:

$$
\left[\begin{array}{ccc}
\frac{1}{16} & \frac{1}{8} & \frac{1}{16} \\
\frac{1}{8} & \frac{1}{4} & \frac{1}{8} \\
\frac{1}{16} & \frac{1}{8} & \frac{1}{16}
\end{array}\right]
$$

We can see that there are two complementary viewpoints for the relation between coarse and fine grids:

1. Coarse grids are used to accelerate the convergence of the smooth components of the fine-grid residuals.

2. Fine girds are used to compute correction terms to the coarse-grid equations, yielding fine-grid accuracy on the coarse girds.

At the coarsest-grid, we have one remaining task before implementing our nonlinear multi-grid algorithm: choosing a nonlinear relaxation scheme. Our first choice is the nonlinear Gauss-Seidel scheme. If the discretized Equation (11) is written with some choice of ordering as:

$$
T_{i}\left(u_{1}, \ldots, u_{N}\right)=z_{i}, i=1, \ldots, N
$$

Then the nonlinear Gauss-Seidel schemes solves:

$$
T_{i}\left(u_{1}, \ldots, u_{i-1}, u_{i}^{\text {new }}, u_{i+1}, \ldots, u_{N}\right)=z_{i}
$$

for $u_{i}^{\text {new }}$. Often Equation is linear in $u_{i}^{\text {new }}$, since the nonlinear terms are discretized by means of its neighbors. If this is not the case, we replace Equation (19) by one step of a Newton iteration:

$$
u_{i}^{\text {new }}=u_{i}^{\text {old }}-\frac{T_{i}\left(u_{i}^{\text {old }}\right)-z_{i}}{\partial T_{i}\left(u_{i}^{\text {old }}\right) / \partial u_{i}}
$$




\section{Experiments}

In this paper, we use the ultrasound images $(256 \times 256)$ to test our method and the algorithm has been implemented using an Intel Pentium IV 1Ghz with 128M RAM, under the Visual $\mathrm{C}++6.0$ environment.

We compared the results of our approach with other speckle reduction techniques including AWMF, Wiener filtering. The quality measurements of mean-square error (MSE) and signal-to-MSE ratio (SMSE) were computed and shown in Table.1.

The MSE is defined as:

$$
M S E=\frac{1}{K} \sum_{i=1}^{K}\left(\tilde{g_{i}}-g_{i}\right)^{2}
$$

where: $\tilde{g}$ is the denoised image, $g$ is the original image and $K$ is image size.

$$
S M S E=10 \log _{10}\left(\sum_{i=1}^{K} g_{i}^{2} / \sum_{i=1}^{K}\left(\tilde{g_{i}}-g_{i}\right)^{2}\right)
$$



(a)



(c)

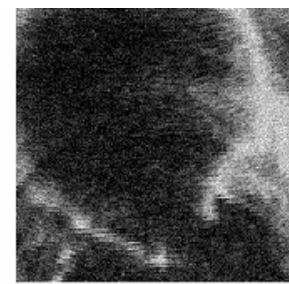

(b)

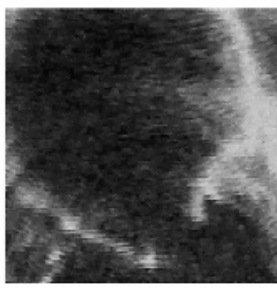

(e)

Fig. 1. Results of various speckle reduction methods. (a) Original mitral valve ultrasound image. (b) Image degraded with simulated speckle noise. (c) AWMF. (d) Wiener filtering. (e) Proposed method.

Table 1. Quality measures obtained by three denoised methods tested on speckled mitral valve ultrasound image at various noise levels

\begin{tabular}{lllllll}
\hline Method & MSE & SMSE & MSE & SMSE & MSE & SMSE \\
\hline Without Filtering & 296 & 13.7 & 125 & 17.4 & 557 & 10.9 \\
AWMF & 134 & 17.2 & 79 & 19.5 & 215 & 15.1 \\
Wiener Filtering & 80 & 19.3 & 53 & 21.2 & 150 & 16.6 \\
Proposed method & 71 & 20.0 & 40 & 22.4 & 122 & 17.5 \\
\hline
\end{tabular}




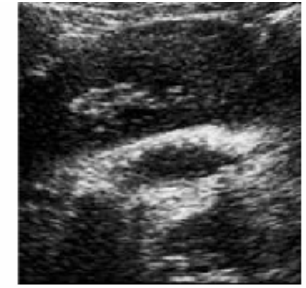

(a)

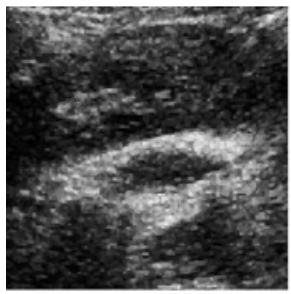

(c)

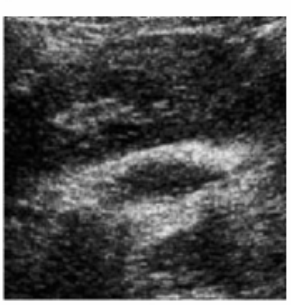

(d)

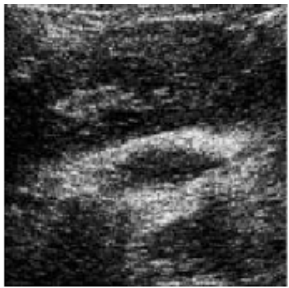

(b)

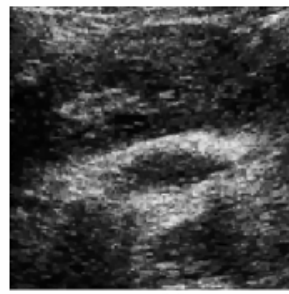

(c)

Fig. 2. Results of various speckle reduction methods. (a) Original kidney ultrasound image. (b) Image degraded with simulated speckle noise. (c) AWMF. (d) Wiener filtering. (e) Proposed method.

Table 2. Time consuming comparison for solving the nonlinear TV-based equation

\begin{tabular}{ccccccc}
\hline $\begin{array}{c}\text { Image } \\
\text { size }\end{array}$ & $256 \times 256$ & $180 \times 180$ & $128 \times 128$ & $80 \times 80$ & $64 \times 64$ & $40 \times 40$ \\
\hline CG & $15.42 \mathrm{~s}$ & $8.20 \mathrm{~s}$ & $3.7 \mathrm{~s}$ & $1.42 \mathrm{~s}$ & $0.63 \mathrm{~s}$ & $0.33 \mathrm{~s}$ \\
$\begin{array}{c}\text { Multi- } \\
\text { Grid }\end{array}$ & $5.77 \mathrm{~s}$ & $2.82 \mathrm{~s}$ & $1.32 \mathrm{~s}$ & $0.55 \mathrm{~s}$ & $0.33 \mathrm{~s}$ & $0.23 \mathrm{~s}$ \\
\hline
\end{tabular}

The denoised images are shown in Fig.1 and Fig.2 for visual comparison. The results show that the TV-based speckle reduction method performs better than the AWMF, as well as the Wiener filter. In addition, from Table. 2 we can see that the nonlinear multi-grid algorithm is more suitable for the nonlinear TV-based minimization problem than the CG method.

\section{Conclusions}

In this paper, a new nonlinear method for speckle suppression in ultrasound images is presented. The main innovation is the use of TV regulation to reduce ultrasound speckle while preserving the appearance of structured regions and organ surfaces. By applying the multi-grid nonlinear algorithm, the technique has the advantage of speed of computation and has a large potential in real-time ultrasound imaging enhancement. 


\section{Acknowledgments}

This work was partially supported by National Science Research Program of China (No. 2004BA714802) and Shanghai Science and Technology Development Foundation (034119820).

\section{References}

1. C.B. Burkhardt, "Speckle in ultrasound B-mode scans," IEEE Trans. Sonics Ultrasonic, 1978(25)(1), 1-6.

2. T. Loupas, W.N. Mcdicken and P.L.Allan, "An adaptive weighted median filter for speckle suppression in medical ultrasonic images," IEEE Trans. Circuits System, 1989(36), 129135.

3. J.C. Bamber and C. Daft, "Adaptive filtering for reduction of speckle in ultrasound pulseecho images," Ultrasonics, 1986, 41-44.

4. S. Gupta, R.C. Chauhan, S.C. Sexana, "Wavelet-based statistical approach for speckle reduction in medical ultrasound images," IEE Med.Biol.Eng.Comput. 2004(42),189-192.

5. L. Rudin, S. Osher and E. Fatemi, "Nonlinear total variation based noise removal algorithms," Physica D, 1992(60), 259-268.

6. V. Dutt, "Statistical analysis of ultrasound echo envelope," Ph.D. dissertation, Mayo Graduate School, Rochester, MN, 1995.

7. J.W. Goodman, "Some fundamental properties of speckle", Journal of Opt. Soc. Am, 1976(66), 1145-1150.

8. Curtis. RV, Mary EO, "Fast, robust total variation-based reconstruction of noisy, blurred images", IEEE Transactions on Image processing, 1998 7(6), 813-824.

9. Brandt. A, "In Multi-grid Methods", Springer Lecture Notes in Mathematics, 1982, No. 960. 